\title{
ROLE OF $\gamma$-KAFIRIN IN THE FORMATION AND ORGANIZATION OF KAFIRIN
}

MICROSTRUCTURES

Joseph O. Anyango, John R.N. Taylor, Janet Taylor*

Institute for Food, Nutrition and Well-being and Department of Food Science, University of Pretoria, Private Bag X20, Hatfield 0028, South Africa.

* Corresponding author +27 12420 5402, Fax: +27 124202839

Email: Janet.Taylor@up.ac.za 


\section{ABSTRACT}

The possible importance of the cysteine-rich $\gamma$-prolamin in kafirin and zein functionality has been neglected. Gamma-kafirin's role in organized microstructures was investigated in microparticles. Residual kafirin (total kafirin minus $\gamma$-kafirin) "microparticles" were nondiscrete (amorphous mass of material), as viewed by electron microscopy and atomic force microscopy. Adding $15 \% \gamma$-kafirin to residual kafirin resulted in formation of a mixture of non-discrete material and nanosize discrete spherical structures. Adding 30\% $\gamma$-kafirin to the residual kafirin resulted in discrete spherical nanosize particles. FTIR indicated that $\gamma$-kafirin had a mixture of random coil and $\beta$-sheet conformation, in contrast to total kafirin which is mainly $\alpha$-helical. Gamma-kafirin also had a very high glass transition temperature $\left(\mathrm{T}_{\mathrm{g}}\right)$ $\left(\approx 270^{\circ} \mathrm{C}\right)$. The conformation and high $\mathrm{T}_{\mathrm{g}}$ of $\gamma$-kafirin probably confer structural stability to kafirin microstructures. Due to its ability to form disulfide cross-links, gamma-kafirin appears to be essential to form and stabilize organized microstructures.

KEYWORDS: Kafirin microparticles, $\gamma$-kafirin, disulfide cross-linking, glass transition temperature, secondary structure. 


\section{INTRODUCTION}

There is great interest in the use of kafirin and zein, the prolamin proteins of sorghum and maize, respectively, as functional proteins to make organized bio-plastic structures such as microparticles, ${ }^{1-3}$ bio-plastic films, ${ }^{2,4}$ fibers,${ }^{5-6}$ sponges,${ }^{7}$ scaffolds ${ }^{8}$ and viscoelastic wheatlike doughs. ${ }^{9}$ These structures have potential in bio-medical applications for use as scaffolds for tissue repair, ${ }^{8,10}$ for encapsulation of bio-actives for controlled or delayed release of drugs, ${ }^{11}$ and nutraceuticals. ${ }^{12}$ Potential food applications may include encapsulation of for example, essential oils ${ }^{1}$, or vitamins ${ }^{13}$ and replacement of gluten in gluten-free formulations for celiac sufferers. ${ }^{9,14-15}$ Bio-plastic films may be used as semi-permeable membranes for food coating to extend perishable food shelf-life. ${ }^{16}$

However, currently there are few commercial products. Reasons for this include high cost and inferior and inconsistent functional properties compared to synthetic polymer plastics ${ }^{17}$ and gluten in the case of baked goods. ${ }^{9}$ Improvement of kafirin and zein functionality is challenging due to their complexity, and lack of uniformity. A number of review articles have been published on kafirin and zein composition, structure and functionality. ${ }^{17-19}$ Briefly, they are small, highly folded proteins, which consist of 4 classes $(\alpha-, \beta-, \gamma-$, and $\delta$-), within which are several sub-classes. They are usually considered hydrophobic proteins but have hydrophilic characteristics and are amphiphilic. Functional properties of their biopolymers are strongly affected by water and temperature.

Recent research indicates that protein aggregation plays a major role in the formation of zein and kafirin micro- or nanostructures. ${ }^{17,20-21}$ and that these nano-structures form the basis of the various bio-plastic materials mentioned above. ${ }^{20}$ Wang and Padua ${ }^{21}$ suggested that assembly of $\alpha$-zein nanostructures is driven by zein's amphiphilic properties and begins by 
the protein structure unfolding. Zein's ( $\alpha$-zein) $\alpha$-helical structure unfolds into $\beta$-sheets, followed by side-by-side placement of anti-parallel $\beta$-sheets. These ribbon-like structures then curl into a ring stabilized by hydrophobic interactions and the ring's 'grow' into nanospheres. However, there is inadequate knowledge as to how this happens. Protein secondary structure in terms of $\alpha$-helical and $\beta$-sheet structure seems to play a key, but incompletely understood role. Beta-sheet structures have been shown to be important in the stabilization of zein-based dough systems. ${ }^{14-15}$ Zein contains a similar amount of $\beta$-sheet structure to gluten if mixed at $35^{\circ} \mathrm{C}$ (above its glass transition temperature) and forms a viscoelastic polymer. However, unlike gluten, heat and shear are necessary to maintain this structure. ${ }^{14}$ Stability of the zein dough could be retained with the addition of a small amount of $\beta$-sheet rich co-protein. ${ }^{15}$ Thus, the viscoelastic properties and relaxation rate of the zein polymers appear to be related to stable $\beta$-sheet structures.

The effects of the presence of the other prolamin classes, in particular $\gamma$-zein or $\gamma$-kafirin, with their high cysteine contents and subsequent ability to form intermolecular disulfide bonds during microstructure formation is of importance. It has been suggested that disulfide bonding through cysteine residues could stabilize kafirin microstructures, ${ }^{22-23}$ in a similar order to that proposed for the stabilization of kafirin and zein protein bodies. ${ }^{24-25}$ but in noncellular system. For zein, the specific zein classes and order in which they are assembled is vital for normal spherical protein bodies to form. ${ }^{25}$ Gamma-zein synthesis is important for initiating protein body formation, retention of $\alpha$-zein within the protein body and maintenance of the spherical orientation of the protein bodies. ${ }^{25}$ Transgenic sorghum lines with down regulation of synthesis of various combinations of kafirin classes have resulted in protein bodies with varying degrees of deformity. ${ }^{26-29}$ 
It also appears likely that the different prolamin classes may play specific roles in the formation and functionality of kafirin and zein structures. Schober et $\mathrm{al}^{30}$ found that zein, which was predominantly $\alpha$-zein, with approximately $10 \%$ co-extracted $\beta$ - and $\gamma$-zein was the best for aggregation in warm water to produce a gluten-like material, whereas film formation was less sensitive to the presence of $\beta$ - and $\gamma$-zein.

Using kafirin microparticles as an example of a prolamin bio-plastic structure, this study describes the role of $\gamma$-kafirin class in their formation and organization. Microparticles is a term used to collectively refer to microcapsules (single core surrounded by a layer of wall material) or microspheres (core dispersed in a continuous matrix network) with a size range of $1-250 \mu \mathrm{m} .^{31}$ The information will be useful in the production of different forms of prolamin microstructures with improved functionality for various different applications including biomaterials and gluten-like networks for viscoelastic dough.

\section{MATERIALS AND METHODS}

\section{Materials}

Total kafirin was extracted from decorticated white, tan-plant non-tannin sorghum grain as described. $^{32}$ Gamma-kafirin was isolated from total kafirin using $0.05 \mathrm{M}$ sodium lactate containing $2 \%(\mathrm{v} / \mathrm{v}) 2$-mercaptoethanol ${ }^{33-34}$ at a protein to solvent ratio of 1:5. Briefly, total kafirin was mixed with a solution containing 2\% (v/v) 2-mercaptoethanol in $0.05 \mathrm{M}$ sodium lactate for $1 \mathrm{~h}$ at $25^{\circ} \mathrm{C}$ with constant stirring. The mixture was centrifuged at $7200 \mathrm{~g}$ for 10 min and the supernatant was collected. The extraction process was repeated twice on the residual pellet and the supernatants bulked. The supernatant was dialyzed against distilled water for $36 \mathrm{~h}$ at $10^{\circ} \mathrm{C}$ using dialysis tubing with a $12-14 \mathrm{kDa}$ cut off (Visking ex Labretoria, 
Pretoria, South Africa) with frequent changes of water. The dialyzed material ( $\gamma$-kafirin) and residual pellet (referred to as residual kafirin) were freeze dried.

\section{Preparation of kafirin microparticles}

Microparticles were prepared by simple coacervation which is a controlled precipitation of a polymeric solution by adding an incompatible solvent. ${ }^{35}$ Microparticles were produced from a solution of kafirin or residual kafirin with $0 \%, 15 \%$ and $30 \%$ (as a proportion of total kafirin protein content) $\gamma$-kafirin added back, in glacial acetic acid according to Taylor et $\mathrm{al}^{3}$ as modified. ${ }^{36}$ This resulted in microparticles suspended in $5.4 \%$ acetic acid.

\section{SDS-PAGE}

Kafirin samples and microparticles made from them, were characterized using SDS-PAGE under non-reducing and reducing conditions on pre-prepared 4-12\% BT gradient gels (Invitrogen Life Technologies, Carlsbad, CA) using an X Cell SureLock Mini-Cell electrophoresis unit (Invitrogen Life Technologies). Kafirin microparticles were washed three times with distilled water to remove the acetic acid prior to SDS-PAGE. The protein loading was $\approx 10 \mu \mathrm{g}$. Invitrogen Mark12 Unstained Standard was used. Protein bands were stained with Coomassie Brilliant Blue R-250 and photographed using a flat-bed scanner.

\section{Microscopy}

\section{Electron microscopy}

For high resolution scanning electron microscopy (SEM) imaging, the microparticle suspensions in distilled water were pipetted on microscope cover slips mounted on an aluminium stub, air-dried, coated with carbon and viewed with a Zeiss Ultra-Plus 55 FEGSEM (Oberkochen, Germany) using an accelerating voltage of $1 \mathrm{kV}$. Transmission electron 
microscopy (TEM) samples were prepared by pipetting the microparticle suspensions directly onto copper micro-grids coated with carbon film and air dried. Viewing was by a JEM-2100F Field Emission Electron Microscope (Jeol, Tokyo, Japan) with an accelerating voltage of 200 $\mathrm{kV}$.

\section{Atomic force microscopy}

Microparticle suspensions in distilled water were pipetted on mica and air-dried. Samples were viewed with a Veeco Icon Dimension Atomic Force Microscope (Bruker, Cambridge, United Kingdom) using ScanAsyst (peak force tapping) mode. ${ }^{37}$ A 2 nm silicon tip on nitride lever cantilever was used.

\section{Fourier transform infrared (FTIR) spectroscopy}

Protein secondary structures were determined by FTIR spectroscopy as described ${ }^{3}$. Freezedried kafirin microparticles were further dried in a desiccator containing silica gel for $72 \mathrm{~h}$ and then scanned using a Vertex 70v FT-IR spectrophotometer (Bruker Optik, Ettlingen,

Germany), using 64 scans, $8 \mathrm{~cm}^{-1}$ band width and an interval of $1 \mathrm{~cm}^{-1}$ in the Attenuated Total Reflectance (ATR) mode in wavenumber range $600-4000 \mathrm{~cm}^{-1}$. The FTIR spectra were normalized and Fourier-deconvoluted using Lorentzian filter with a resolution enhancement factor of 2 and $8 \mathrm{~cm}^{-1}$ band width.

\section{Differential scanning calorimetry (DSC)}

Thermal analysis was performed by DSC using a Mettler Toledo HP DSC827 (Schwerzenbach, Switzerland). Freeze-dried samples were further dried in a desiccator containing silica gel for 14 days. Samples approximately $15 \mathrm{mg}$ were accurately weighed into $100 \mu \mathrm{L}$ aluminum pans and sealed immediately. Calibration was based on pure indium. An empty pan was used as reference. DSC scans were performed from $25-280^{\circ} \mathrm{C}$ and heating 
rate of $10^{\circ} \mathrm{C} / \mathrm{min}$ under nitrogen (40 bar pressure). Glass transition $\left(\mathrm{T}_{\mathrm{g}}\right)$ peaks were taken at the peak of step change in heat flow during heating and determined using $\operatorname{STAR}^{\mathrm{e}}$ software Version $9.20 .^{38-39}$

\section{Statistical analysis}

Experiments were repeated at least once, except for FTIR and DSC which were repeated three times. Data were analyzed by one-way analysis of variance (ANOVA). Significant differences among the means were determined by Fischer's least significant difference (LSD) test $(\mathrm{p}<0.05)$

\section{RESULTS AND DISCUSSION}

\section{SDS-PAGE of kafirin proteins}

SDS-PAGE under non-reducing conditions of the isolated $\gamma$-kafirin revealed that it had a major $\gamma$-kafirin band (approx. $28 \mathrm{kDa}$ ) and a fainter band (approx. $46 \mathrm{kDa}$ ), equivalent to the minor $\gamma$-kafirin band (approx. $49 \mathrm{kDa}$ ) described by Evans et al, ${ }^{33}$ both of which have been assigned to $\gamma$-kafirin class. ${ }^{40}$ Additionally, there were disulfide cross-linked oligomers of increasing molecular size (Figure 1, gel A lane 3). The residual kafirin did not show the 28 $\mathrm{kDa} \gamma$-kafirin band or $46 \mathrm{kDa}$ band (gels A and B lane 2). The total kafirin (gels A and B lane 1) differed from the residual kafirin in that it exhibited a more intense monomer band, indicative of the presence of $\gamma$-kafirin. Thus, the $\gamma$-kafirin extraction was effective.

\section{Effect of $\gamma$-kafirin on the morphology of kafirin "microparticles"}

When viewed at low magnification, SEM of "microparticles" prepared using residual kafirin appeared as an amorphous mass and lacked discrete shapes (Figure 2B), indicating failure to organize to the same degree as the total kafirin microparticles (Figure 2A). Higher 
magnification of residual kafirin "microparticles" showed similar structures to those contained within the larger total kafirin microparticles. Adding $15 \% \gamma$-kafirin to the residual kafirin resulted in a mixture of non-discrete material and some nanosize discrete spherical structures (Figure 2C). These nanosize particles were not vacuolated, as observed with TEM (see inset picture in Figure 2C). Adding a higher proportion (30\%) of the $\gamma$-kafirin to the residual kafirin resulted in the formation of discrete spherical nanosize particles (Figure 2D). These particles were smaller in size than control total kafirin microparticles, despite the fact that the level of $\gamma$-kafirin added was higher than the theoretical 9-12\% proportion of $\gamma$-kafirin in total kafirin in vitreous (corneous) and 19-21\% in opaque sorghum grain endosperm, respectively. ${ }^{41}$ The mainly submicron-sized particles also appeared to be made up of spherical nanostructures as viewed with high resolution SEM (see inset pictures in Figure 2). As with the SEM, AFM showed that all kafirin microparticles had a rough surface morphology composed of nanosize protuberances (Figures 3A-D). Similar structures have been reported for kafirin microparticles. ${ }^{36}$ AFM of the surface of these nanosize protuberances at higher magnification showed that adding $\gamma$-kafirin to residual kafirin, especially at a higher level (30\%) resulted in discrete nanoparticles (Figure 3D). This agrees with morphological observation with the SEM (Figure 2D).

Addition of $15 \% \gamma$-kafirin to total kafirin also resulted in relatively smaller microparticles with smoother surface, which appeared to be connected at the edges (marked with ' $\mathrm{X}$ ') (Figure 4B). These connections may have been made up of cross-linked $\gamma$-kafirin forming a layer on already formed microparticles. There was loss of the spherical microparticle structure with addition of $30 \% \gamma$-kafirin to total kafirin (Figure $4 \mathrm{C}$ ). This may be due to massive cross-linking and encasing of the kafirin microparticles within the $\gamma$-kafirin layer, resulting in loss of the spherical structures. An attempt to prepare microparticles from isolated $\gamma$-kafirin using the simple coacervation technique did not yield microparticles (data 
not shown). This was probably because isolated $\gamma$-kafirin is water soluble. ${ }^{33}$ Hence, the protein could not be precipitated out in the simple coacervation technique applied.

From these microscopy data it seems that removal of the cysteine-rich $\gamma$-kafirin from total kafirin prevents formation of spherical kafirin microparticles. Possibly, $\gamma$-kafirin is needed to stabilize the other $\alpha$-, $\beta$-, and $\delta$ - kafirin classes which may be centrally located within the microparticle. Without this, the structure of the microparticles would be destabilized, as demonstrated. This suggestion is based on a model for kafirin microparticles formation ${ }^{17}$ using an analogy of protein body formation but in an isolated and non-cellular system. The model describes that the microparticles form by precipitation of $\alpha$ - and $\beta$-kafirin around small particles of undissolved kafirin and then the $\gamma$-kafirin forms a stabilizing layer on the surface of the partially formed microparticle. Loss of spherical shape has been observed with both kafirin and zein protein bodies, where down regulating the synthesis of the $\gamma$-prolamin class resulted in protein bodies with irregular morphology. ${ }^{25-29,42}$ It has been suggested that the $\gamma$ prolamin plays a role of encasing the other prolamin classes through disulfide crosslinking, thereby preventing formation of irregular-shaped protein bodies. ${ }^{42}$ The fact that the microparticles prepared from residual kafirin with $\gamma$-kafirin added were far smaller than total kafirin microparticles suggests that the interaction of the pre-isolated kafirin proteins may not be optimal compared to innate $\gamma$-kafirin in total kafirin. This is probably because the process of isolating the different kafirin classes may have induced changes to these proteins, as was indicated from the SDS-PAGE of the $\gamma$-kafirin (Figure 1). In terms of kafirin microparticle formation and organization, it seems that, the $\gamma$-kafirin together with $\beta$-kafirin could initiate organization of the kafirin microparticles, while the other classes (primarily $\alpha$-kafirin) may enlarge the microparticles. The fact that these kafirin microparticles appear to be made up of spherical nanoparticles agrees with the findings by Wang and Padua ${ }^{21}$ who worked on zein microspheres prepared by evaporation induced self-assembly. According these authors, a 
sphere seems to be the basis for of all other micro phases produced by self-assembly of zein, kafirin's homologue.

The nano-sized particles prepared using residual kafirin with 30\% $\gamma$-kafirin added, the higher resolution AFM of total kafirin microparticles did not show distinct nanostructures. It seems that pre-isolation of $\gamma$-kafirin and then adding it to the residual kafirin may have resulted in uneven distribution of the $\gamma$-kafirin in the resultant microstructure. The residual kafirins could have already interacted with each other prior to commencement of microparticle formation. This was indicated by SEM of microparticles prepared using total kafirin with added $\gamma$ kafirin, where the $\gamma$-kafirin appeared to form a layer on the surface of the microparticles. An uneven distribution of the $\gamma$-kafirin which encases the residual kafirin classes would probably result in a clear demarcation. The demarcations would be created by the $\gamma$-kafirin layer occurring on the surface of each of the nanostructures that constitute the microparticle. Alternatively, during formation of microparticles with total kafirin all the kafirin classes could interact at virtually the same time. Hence the distribution of the $\gamma$-kafirin in the total kafirin microparticle would be more even, resulting in less distinct boundaries between the nano-sized structures.

An unresolved finding from this study is that while commercial zein, which is essentially only $\alpha$-zein, ${ }^{43}$ can form distinct spherical microparticles ${ }^{44}$ in the absence of the $\gamma$-prolamin class, kafirin did not form discrete microparticles. This suggests that other factors besides the absence of the $\gamma$-prolamin class are involved. Firstly, it is known that commercial zeins are variable ${ }^{45}$ hence may contain small amounts of the other zein classes. Secondly, it has been proposed that $\alpha$-zein has lutein at the core of its triple helical segments, ${ }^{46}$ which helps to stabilize the protein configuration ${ }^{46}$ thereby could stabilize $\alpha$-zein microparticles.

\section{Effects of $\gamma$-kafirin on the chemical structure of kafirin "microparticles"}


SDS-PAGE under non-reducing conditions of the kafirin "microparticles" showed that there was an increase in oligomer bands as a result of addition of $\gamma$-kafirin to the residual kafirin (Figure 5, gel A). A band $\approx 50 \mathrm{kDa}$, which is likely to be a dimer of $\alpha-(\approx 22 \mathrm{kDa})$ and $\gamma$ kafirin $(\approx 28 \mathrm{kDa})^{40}$ was noted for microparticles prepared using residual kafirin with added $\gamma$ kafirin (Figure 5, gel A lanes 3 and 4). However, the oligomer bands were faint under reducing conditions, indicating that the polymerization as a result of addition of $\gamma$-kafirin was due to disulfide cross-linking and that the linkages were broken by the reducing agent, as demonstrated by the increase in intensity of kafirin monomeric bands (Figure 5, gel B lanes 3 and 4). There were no dimer and oligomer bands in the absence of $\gamma$-kafirin with SDS-PAGE of residual kafirin microparticles under reducing conditions (Figure 5, gel B lane 2). This shows the importance of $\gamma$-kafirin in polymerization of kafirin proteins. In contrast, kafirin dimers resistant to reduction by mercaptoethanol were observed with microparticles prepared using either total kafirin or residual kafirin with $\gamma$-kafirin added (Figure 5, gel B lanes 1, 3 and 4). Similar reduction resistant bands were observed with kafirin microparticle films. ${ }^{47}$ It has been suggested that the presence of polymeric kafirin resistant to reduction could be due to some covalent crosslinks being inaccessible to the reducing agent. ${ }^{19}$

Isolated $\gamma$-kafirin showed two peaks in the Amide I region as determined by FTIR with normalization and resolution enhancement using Fourier-deconvolution (Figure 6A). The higher peak at $1643 \mathrm{~cm}^{-1}$ was assigned to random coil and the lower peak at $1628 \mathrm{~cm}^{-1}$ assigned to anti-parallel $\beta$ - sheet. ${ }^{48-50}$ It is possible that the $\beta$-sheet component could contain some polyproline II (PPII) conformation, as the polyproline II (PPII) cannot be measured by solid state FTIR since the $\beta$-sheet and PPII signals overlap. ${ }^{51}$ Gamma-kafirin and $\gamma$-zein both have repeats of a conserved hexapeptide motif (PPPVHL) in the N-terminal domain ${ }^{18}$ and so $\gamma$-kafirin may be expected to exhibit some PPII conformation, as has been reported for $\gamma$ zein. ${ }^{51}$ However, Bansal et $\mathrm{al}^{52}$ using a PSIPRED protein structure prediction server for $\gamma$ - 
kafirin also reported a large proportion of coil (82.79\%), with a small amount of helix $(11.83 \%)$ and extended strand $(5.38 \%)$ but no $\beta$-sheet. In contrast, secondary structure of $\gamma-$ zein has been shown to contain $33 \%$ helix and $31 \% \beta$-sheet when analysed in the solid state by FTIR. $^{53}$

FTIR spectra of kafirin microparticles and residual kafirin 'microparticles' both showed two peaks at wavenumbers between 1653-1655 and 1622-1624 in the Amide 1 region (Figure 6B), which can be assigned to $\alpha$-helical conformation and anti-parallel $\beta$-sheet, respectively. ${ }^{48}$ However, the ratio of $\alpha$-helical to $\beta$-sheet differed. For the total kafirin microparticles this ratio was 1.2 , which was similar to the 1.3 reported by Taylor et al $^{3}$ for kafirin microparticles. The ratio of $\alpha$-helical to $\beta$-sheet for residual kafirin 'microparticles', 1.06, indicated a greater proportion of $\beta$-sheet. Adding $\gamma$-kafirin to the residual kafirin and using the resultant kafirin mixture to prepare microparticles indicated a further small decrease in the ratio of $\alpha$-helical to $\beta$-sheet to 0.99 and 0.98 when $15 \%$ and $30 \% \gamma$-kafirin were added back, respectively. Increase in the relative proportion of $\beta$-sheet structures has been associated with disulfide cross-linking of kafirin proteins. ${ }^{48}$

\section{Effect of $\gamma$-kafirin on the thermal properties of kafirin "microparticles"}

DSC showed $\gamma$-kafirin had significantly higher $T_{g}(p<0.05)$ compared to total kafirin and residual kafirin (Figure 7A). This may be due to $\gamma$-kafirin's high cysteine residue content, about $7 \mathrm{~mol} \%,{ }^{18}$ resulting in a greater extent of disulfide cross-linking. To the best of the authors' knowledge, this is also the first report of $\mathrm{T}_{\mathrm{g}}$ of $\gamma$-kafirin. Adding $\gamma$-kafirin to residual kafirin increased the kafirin microparticle $\mathrm{T}_{\mathrm{g}}$ significantly $(\mathrm{p}<0.05)$ (Figure $\left.7 \mathrm{~B}\right)$. The increase in the $\mathrm{T}_{\mathrm{g}}$ was likely also due to increase in disulfide cross-linking. ${ }^{54}$ The microparticles had significantly higher $\mathrm{T}_{\mathrm{g}}$ (Figure $\left.7 \mathrm{~B}\right)(\mathrm{p}<0.05)$ compared to proteins from which they were prepared (Figure 7A), probably because of increase in relative proportion of the $\beta$-sheet 
conformations during microparticle formation. As $\beta$-sheet conformations are associated with protein unfolding, ${ }^{48}$ alignment of these protein molecules may result in an increase in disulfide cross-linking or hydrogen bonding, thereby increasing the $\mathrm{T}_{\mathrm{g}}$. The $\mathrm{T}_{\mathrm{g}}$ value for total kafirin in the present study $\left(\approx 248^{\circ} \mathrm{C}\right)$ was higher than the $\approx 230^{\circ} \mathrm{C}$ reported by Wang et al, ${ }^{55}$ possibly due to differences in moisture contents. In the present study the freeze-dried samples were dried further in a desiccator for over two weeks, while in the Wang et $\mathrm{al}^{55}$ study the samples were not further dried. Moisture content is known to strongly affect the $T_{g}$ of prolamin proteins ${ }^{56-58}$ by acting as a plasticizer.

This work has demonstrated that the $\gamma$-kafirin class is required to form spherical kafirin microstructures (microparticles). It stabilizes their structure due to its ability to form disulfide crosslinks, probably encasing the other kafirin classes in a similar manner to that which occurs in prolamin protein bodies. ${ }^{42}$ Adding $\gamma$-kafirin to total kafirin does not result in larger particles as the excess cross-linking by the $\gamma$-kafirin seems to create an imbalance among the forces that maintain the spherical microparticle shape thereby destabilizing the microparticle structure. The work has presented for the first time $T_{g}$ data of isolated $\gamma$-kafirin, which shows that its $\mathrm{T}_{\mathrm{g}}$ is higher than that of total kafirin and residual kafirin. This improved understanding on the role of $\gamma$-prolamin class in kafirin microstructures and knowledge on the secondary structure and thermal properties of isolated $\gamma$-kafirin will be useful in the development of kafirin or zein bio-plastics with functional properties that are more comparable to synthetic polymer plastics.

\section{FUNDING}

Joseph Anyango is grateful for the provision of a University of Pretoria Postdoctoral Fellowship. 
We thank A. Hall, C. van der Merwe, A. Botha and A. Buys for their assistance with microscopy.

\section{ABBREVIATIONS USED}

SDS-PAGE, sodium dodecyl sulfate-polyacrylamide gel electrophoresis; SEM, scanning electron microscopy; TEM, transmission electron microscopy; AFM, atomic force microscopy; DSC, differential scanning calorimetry; FTIR, Fourier transform infrared spectroscopy, $\mathrm{T}_{\mathrm{g}}$, glass transition temperature.

\section{REFERENCES}

(1) Parris, N.; Cooke, P.H.; Hicks, K.B. Encapsulation of essential oils in zein nanospherical particles. J. Agric. Food Chem. 2005, 53, 4788-4792.

(2) Wang, H.J.; Lin, Z.X.; Liu, X.M.; Sheng, S.H.; Wang, J.Y. Heparin-loaded zein microsphere film and hemocompatibility. J. Controlled Release 2005, 105, 120-131.

(3) Taylor, J.; Taylor, J.R.N.; Belton, P.S.; Minnaar, A. Formation of kafirin microparticles by phase separation from an organic acid and their characterisation. J. Cereal Sci. 2009, 50, 99-105.

(4) Gao, C.; Taylor, J.; Wellner, N.; Byaruhanga, Y.B.; Parker, M.L.; Mills, E.N.C.; Belton, P.S. Effect of protein secondary structure and biofilm formation of kafirin. J. Agric. Food Chem. 2005, 52, 2382-2385.

(5) Torres-Giner, S.; Gimenez, E.; Lagaron, J.M. Characterization of the morphology and thermal properties of zein prolamin nanostructures obtained by electrospinning. Food Hydrocoll. 2008, 22, 601-614. 
(6) Wang, Y.; Chen, L. Electrospinning of prolamin proteins in acetic acid: the effects of protein conformation and aggregation in solution. Macromol. Mater. Eng. 2012, 297, 902-913.

(7) Padua, G.W.; Wang, Q.; Wang, Y. Construction and properties of zein-based cell supports and scaffolds. NSTI-Nanotech. 2010, 3, 202-205.

(8) Gong, S.; Wang, H.; Sun, Q.; Xue, S.-T.; Wang, J.-Y. Mechanical properties and in vitro biocompatibility of porous zein scaffolds. Biomaterials 2006, 27, 3793-3799.

(9) Erickson, D.P.; Campanella, O.H.; Hamaker, B.R. Functionalizing maize zein in viscoelastic dough systems through fibrous, $\beta$-sheet-rich protein networks: An alternative, physicochemical approach to gluten-free breadmaking. Trends Food Sci. Technol. 2012, 24, 74-81.

(10) Tu, J.; Wang, H.; Li, H.; Wang, J.; Zhang, X. The in vivo bone formation by mesenchymal stem cells in zein scaffolds. Biomaterials 2009, 30, 4369-4376.

(11) Liu, X., Sun, Q., Wang, H., Zhang, J.Y. Microspheres of corn protein, zein, for ivermectin drug delivery system. J. Controlled Release 2005, 26, 102-131.

(12) Taylor, J.; Taylor, J.R.N.; Belton, P.S.; Minnaar, A. Kafirin microparticle encapsulation of catechin and sorghum condensed tannins. J. Agric. Food Chem. 2009, 57, 7523-7528.

(13) Luo, Y.; Teng, Z.; Wang, Q. Development of zein nanoparticles coated with carboxymethyl chitosan for encapsulation and controlled release of vitamin D3. J. Agric. Food Chem. 2012, 60, 836-843.

(14) Mejia, C.D.; Mauer, L.J.; Hamaker, B.R. Similarities and differences in secondary structure of viscoelastic polymers of maize $\alpha$-zein and wheat gluten proteins. J. Cereal Sci. 2007, 45, 353-359. 
(15) Mejia, C.D.; Gonzalez, D.C.; Mauer, L.J.; Campanella, O.H.; Hamaker, B.R. Increasing and stabilizing $\beta$-sheet structure of maize zein causes improvement in its rheological properties. J. Agric. Food Chem. 2012, 60, 2316-2321.

(16) Buchner, S.; Kinnear, M.; Crouch, I.J.; Taylor, J.; Minnaar, A. Extending the postharvest sensory quality and shelf-life of 'Packham's Triumph' pears with a kafirin protein coating. J.Sci. Food Agric. 2011, 91, 2814-2820.

(17) Taylor, J.; Anyango, J.O.; Taylor, J.R.N. Developments in the science of zein, kafirin and gluten protein bio-plastic materials. Cereal Chem. 2013, 90, 344-357.

(18) Belton, P.S.; Delgalligo, I.; Halford, N.G.; Shewry, P.R. Kafirin structure and functionality. J. Cereal. Sci. 2006, 44, 272-286.

(19) Duodu, K.G.; Taylor, J.R.N.; Belton, P.S.; Hamaker, B. R. Factors affecting sorghum protein digestibility. J. Cereal Sci. 2003, 38, 117-131.

(20) Wang, Y.; Padua, G.W. Formation of zein microphases in ethanol-water. Langmuir 2010, 26:12897-12901.

(21) Wang, Y.; Padua, G.W. Nanoscale characterization of zein self-assembly. Langmuir 2012, 28, 2429-2435.

(22) Taylor, J. Preparation, characterisation and functionality of kafirin microparticles. PhD thesis, University of Pretoria, Pretoria, South Africa. 2008.

(23) Anyango, J.O. Physico-chemical modification of kafirin microstructures for application as biomaterials. PhD thesis, University of Pretoria, Pretoria, South Africa. 2012.

(24) Shull, J.M.; Watterson, J.J.; Kirleis, A.W. Purification and immunocytochemical localisation of kafirins in Sorghum bicolor (L. Moench) endosperm. Protoplasma 1992, $171,64-74$. 
(25) Coleman, C.E.; Herman, E.M.; Takasaki, K.; Larkins, B.A. The maize $\gamma$-zein sequesters $\alpha$-zein and stabilizes its accumulation in protein bodies of transgenic tobacco endosperm. Plant Cell 1996, 8, 2335-2345.

(26) Wu, Y.; Holding, D.R.; Messing, J. Gamma-zeins are essential for endosperm modification in quality protein maize. Proc. Natl. Acad. Sci. U.S.A. 2010, 107, 1281012815.

(27) Da Silva, L.S.; Jung, R.; Zhao, Z.; Glassman, K.; Taylor, J.; Taylor, J.R.N. Effect of suppressing the synthesis of different kafirin sub-classes on grain endosperm texture, protein body structure and protein nutritional quality in improved sorghum lines. J. Cereal Sci. 2011, 54, 160-167.

(28) Da Silva, L.S.; Taylor, J.; Taylor, J.R.N. Transgenic sorghum with altered kafirin synthesis: kafirin solubility, polymerization and protein digestion. J. Agric. Food Chem. 2011, 59, 9265-9270.

(29) Kumar, T.; Dweikat, I.; Sato, S.; Ge, Z.; Nersesian, N.; Chen, H.; Elthon, T.; Bean, S.; Ioerger, B.P.; Tilley, M.; Clemente, T. Modulation of kernel storage proteins in grain sorghum (Sorghum bicolor (L.) Moench). Plant Biotech. J. 2012, 10, 533-544.

(30) Schober, T.J., Bean, S.R., Tilley, M., Smith, B.M., Ioerger, B.P. Impact of different isolation procedures on the functionality of zein and kafirin. J. Cereal Sci. 2011, 54, 241249.

(31) Reis, C.P.; Neufeld, R.J.; Ribeiro, A.J.; Veiga, F. Nanoencapsulation II. Biomedical applications and current status of peptide and protein nanoparticulate delivery systems. Nanomed. Nanotechnol. Biol. Med. 2006, 2, 53-65.

(32) Emmambux, N.M.; Taylor, J.R.N. Sorghum kafirin interaction with various phenolic compounds. J. Sci. Food Agric. 2003, 83, 402-407. 
(33) Evans, D.J.; Schüssler, L.; Taylor, J.R.N. Isolation of reduced-soluble protein from sorghum starchy endosperm. J. Cereal Sci. 1987, 5, 61-67.

(34) Taylor, J.; Bean, S.R.; Ioerger, B.P.; Taylor, J.R.N. Preferential binding of sorghum tannins with g-kafirin and the influence of tannin binding on kafirin digestibility and biodegradation. J. Cereal Sci. 2007, 46, 22-31.

(35) Mathiowitz, E.; Kreitz, M.R.; Brannon-Peppas, L. Microencapsulation. In Encyclopedia of Controlled Drug Delivery, Mathiowitz, E., Ed.; John Wiley \& Sons Inc.: New York, NY, 1999; Vol. 1, pp. 493-546.

(36) Anyango, J. O.; Duneas, N.; Taylor, J.R.N.; Taylor, J. Physicochemical modification of kafirin microparticles and their ability to bind bone morphogenetic protein-2 (BMP-2), for application as a biomaterial. J. Agric. Food Chem. 2012, 60, 8419-8426.

(37) Kaemmer, S.B. Introduction to Bruker's ScanAsyst and PeakForce tapping AFM technology. Bruker Application Note \#133, Bruker Nano Inc.: Santa Barbara, CA, 2011.

(38) Thermal Analysis UserCom 11. Interpreting DSC curves. Part 1: Dynamic measurements. Information for users of Mettler Toledo thermal analysis system. 1/2000. (http://uk.mt.com/dam/non-indexed/po/ana/ta-usercom/51710020_UserCom11_TA_e.pdf) (Accessed August 2013).

(39) Wang, Y.; Rakotonirainy, A.M.; Padua, G.W. Thermal behavior of zein-based biodegradable films. Starch/Stärke 2003, 55, 25-29.

(40) El Nour, N.A.; Peruffo, A.D.B.; Curioni, A. Characterisation of sorghum kafirins in relations to their cross-linking behavior. J. Cereal Sci. 1998, 28, 197-207.

(41) Oria, M.P.; Hamaker, B.R.; Axtell, J.D.; Huang, C.-P. A highly digestible sorghum mutant cultivar exhibits a unique folded structure of endosperm protein bodies. Proc. Natl. Acad. Sci. U.S.A. 2000, 97, 5065-5070. 
(42) Wu, Y.; Messing, J. RNA interference-mediated change in protein body morphology and seed opacity through loss of different zein proteins. Plant Physiol. 2010, 153, 337347.

(43) Lawton, J.W. Zein: a history of processing and use. Cereal Chem. 2002, 79, 1-18.

(44) Zhong, Q.; Jin, M. Zein nanoparticles produced by liquid-liquid dispersion. Food Hydrocoll. 2009, 23, 2380-2387.

(45) Selling, G.W.; Lawton, J.; Bean, S.; Dunlap, C.; Sessa, D.J.; Willett, J. L.; Byars, J. Rheological studies utilizing various lots of zein in $N, N$-dimethylformamide solutions. J. Agric. Food Chem. 2005, 53, 9050-9055.

(46) Momany, F.A.; Sessa, D.J.; Lawton, J.W.; Selling, G.W.; Hamaker, S.A.H.; Willet, J.L. Structural Characterization of $\alpha$-Zein. J. Agric. Food Chem. 2006, 54, 543-547.

(47) Anyango, J.O.; Taylor, J.; Taylor, J.R.N. Improvement in water stability and other related functional properties of thin cast kafirin films. J. Agric. Food Chem. 2011, 59, $12674-12682$.

(48) Duodu, K.G.; Tang, H.; Grant, A.; Wellner, N.; Belton, P.S.; Taylor, J.R.N. FTIR and solid state ${ }^{13} \mathrm{C}$ NMR spectroscopy of proteins of wet cooked and popped sorghum and maize. J. Cereal Sci. 2001, 33, 261-269.

(49) Mizutani, Y.; Matsumura, Y.; Imamura, K.; Nakanishi, K.; Mori, T. Effects of water activity and lipid addition on secondary structure of zein in powder systems. J. Agric. Food Chem. 2003, 51, 229-235.

(50) Kong, J.; Yu, S. Fourier transform infrared spectroscopic analysis of protein secondary structures. Acta Biochim. Biophys. Sin. 2007, 39, 579-559.

(51) Bicudo, T.C.; Bicudo, R.C.; Forato, L.A.; Beltramini, L.M.; Batista, L.A.; Filho, R.B.; Colnago, L.A. $\gamma$-Zein secondary structure in solution by circular dichroism. Biopolymers 2008, 89, 175-178. 
(52) Bansal, S.; Mishra, A.; Tomar, A.; Sharma, S.; Khanna, V.K.; Garg, G.K. Isolation and temporal endospermal expression of $\gamma$-kafirin gene of grain sorghum (Sorghum bicolor L. moench) var. M 35-1 for introgression analysis of transgene. J. Cereal Sci. 2008, 48, 808-815.

(53) Bicudo, T.C.; Forato, L.A.; Batista, L.A.R.; Colnago, L.A. Study of the conformation of $\gamma$-zeins in purified maize protein bodies by FTIR and NMR spectroscopy. Anal Bioanal Chem 2005, 383, 291-296.

(54) Matveev, Y.I.; Grinberg, V.Y.; Sochava, I.V.; Tolstoguzov, V.B. Glass transition temperature of proteins. Calculation based on the additive contribution method and experimental data. Food Hydrocoll. 1997, 11, 125-133.

(55) Wang, Y.; Tilley, M.; Bean, S.; Sun, X.S.; Wang, D. Comparison of methods for extracting kafirin proteins from sorghum distillers dried grains with solubles. J. Agric. Food Chem. 2009, 57, 8366-8372.

(56) Lawton, J.W. Viscoelasticity of zein-starch doughs. Cereal Chem. 1992, 69, 351-355.

(57) De Graaf, E.M.; Madeka, H.; Cocero, A.M.; Kokini, J.L. Determination of the effect of moisture on gliadin glass transition using mechanical spectrometry and differential scanning calorimetry. Biotechnol. Prog. 1993, 9, 210-213.

(58) Madeka, H.; Kokini, J.L. Effect of glass transition and cross-linking on rheological properties of zein: development of a preliminary state diagram. Cereal Chem. 1996, 73, 433-438. 


\section{FIGURE CAPTIONS}

Figure 1. SDS-PAGE under non-reducing (A) and reducing conditions (B) of kafirin. Protein loading, $\approx 10 \mu \mathrm{g}$. Lanes MW: Molecular markers, 1: Total kafirin, 2: Residual kafirin, 3: $\gamma$ kafirin.

Figure 2. Effects of adding $\gamma$-kafirin to residual kafirin on the morphology of air-dried kafirin microparticles as examined by electron microscopy. A. Total kafirin microparticles. B. Residual kafirin 'microparticles'. C. Residual kafirin $+15 \% \quad \gamma$-kafirin microparticles. D. Residual kafirin $+30 \% \gamma$-kafirin microparticles. The insets are higher magnifications. SEM Scanning electron microscopy; TEM-Transmission electron microscopy.

Figure 3. Effects of adding $\gamma$-kafirin to residual kafirin on the morphology of air-dried kafirin microparticles as examined by atomic force microscopy (AFM). A. Total kafirin microparticles. B. Residual kafirin 'microparticles'. C. Residual kafirin $+15 \% \gamma$-kafirin microparticles. D. Residual kafirin $+30 \% \gamma$-kafirin microparticles. Higher magnifications of the regions inside the frame are shown at the bottom.

Figure 4. Effects of adding $\gamma$-kafirin to total kafirin on the morphology of air-dried kafirin microparticles as examined by scanning electron microscopy. A. Total kafirin microparticles. B. Total kafirin $+15 \% \gamma$-kafirin microparticles. ' $\mathrm{X}$ ' marks the connection point. $\mathbf{D}$. Total kafirin $+30 \% \gamma$-kafirin microparticles. The bottom images are higher magnifications.

Figure 5. SDS-PAGE under non-reducing (A) and reducing conditions (B) of kafirin microparticles. Protein loading, $\approx 10 \mu \mathrm{g}$. Lanes MW: Molecular markers, 1: Total kafirin, 2: Residual kafirin, 3: Residual kafirin $+15 \% \gamma$-kafirin, 4: Residual kafirin $+30 \% \gamma$-kafirin. 
Figure 6. FTIR spectra of kafirin and air-dried kafirin microparticles. A. Total kafirin, residual kafirin and $\gamma$ - kafirin proteins. B. Kafirin microparticles. Spectra offset for clarity.

Figure 7. Typical DSC thermograms of kafirin proteins and kafirin microparticles. A. Proteins. B. Microparticles. Arrows mark melting temperatures, which are probably $\mathrm{T}_{\mathrm{g}}$. 


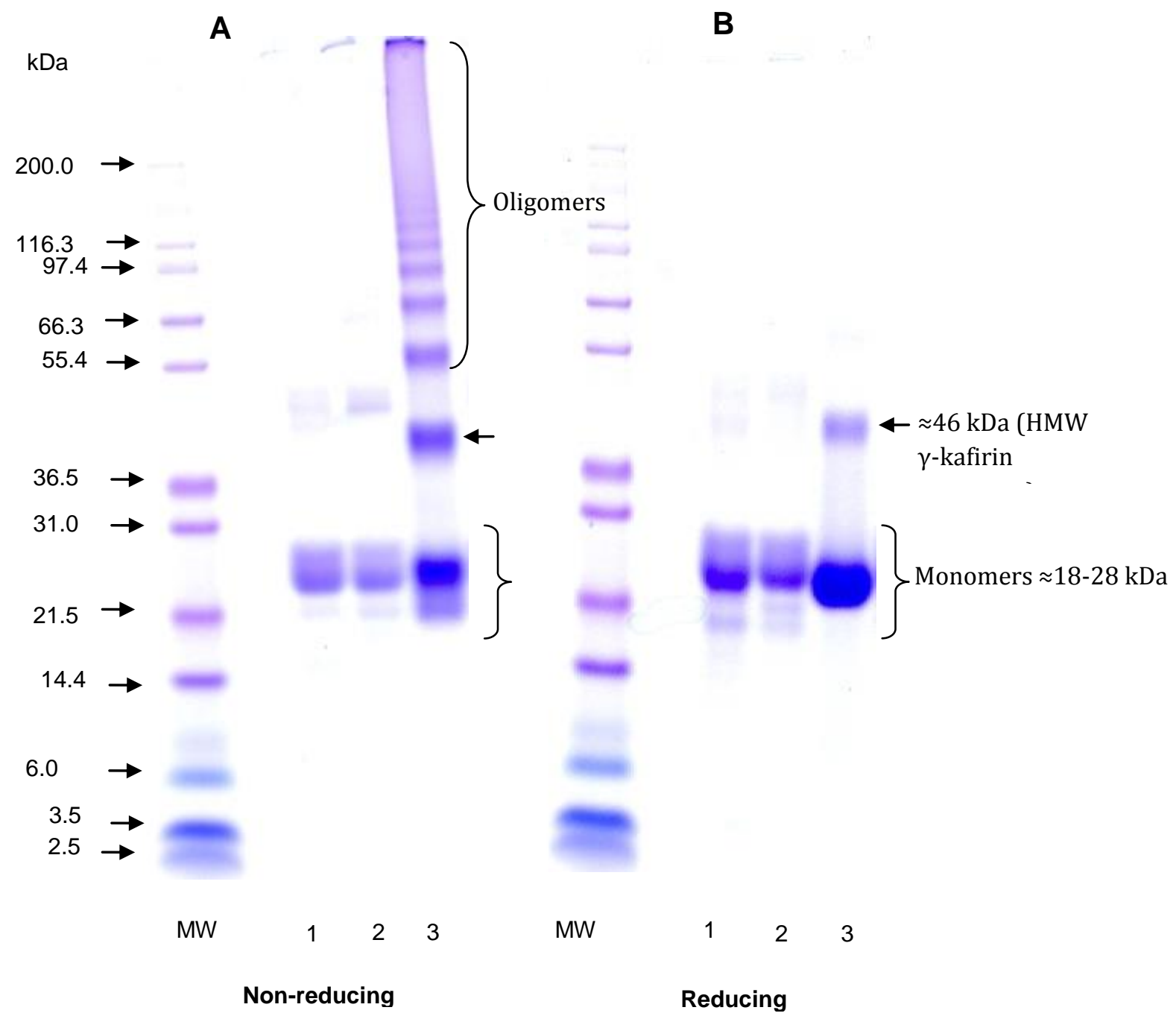

Figure 1 


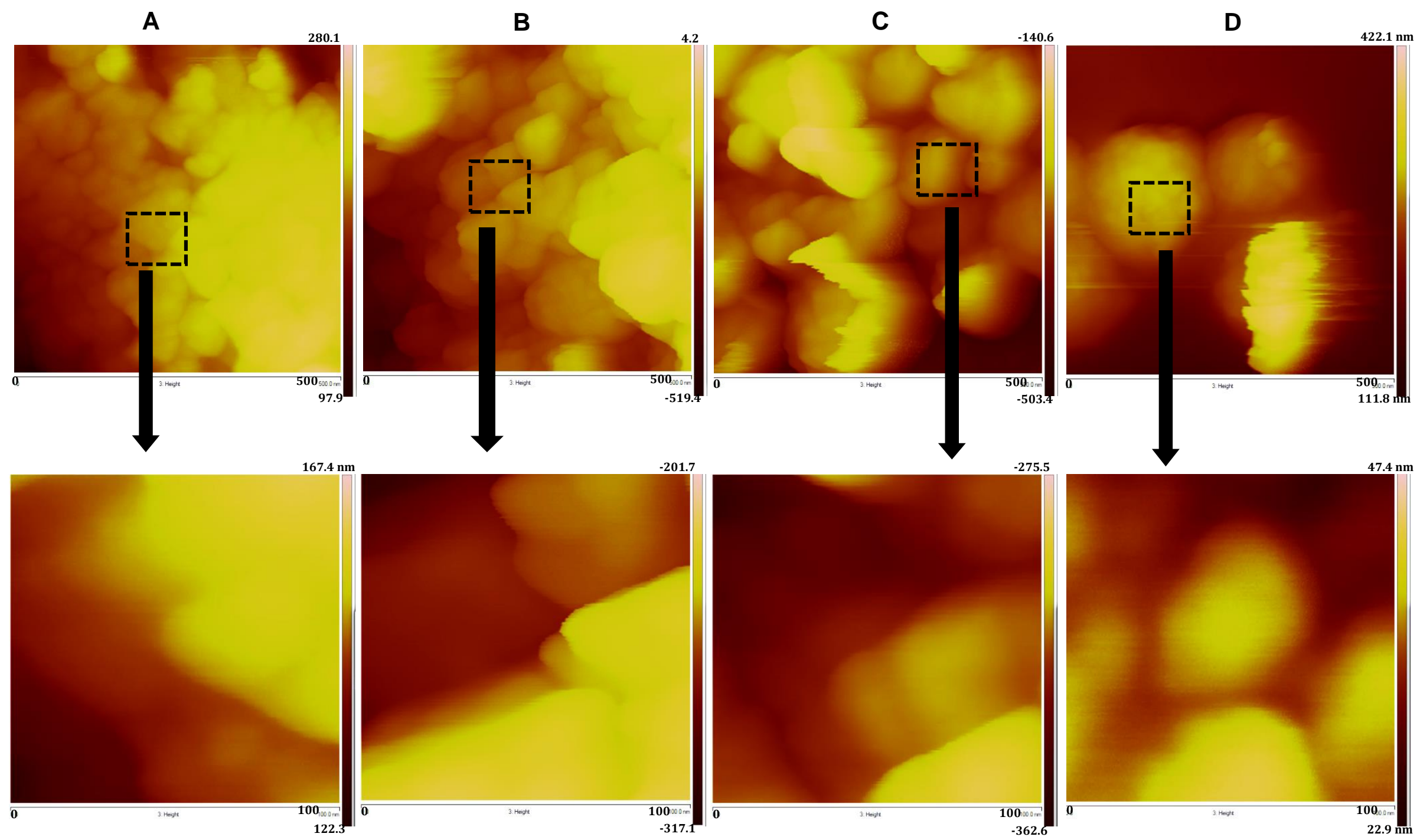

Figure 3 


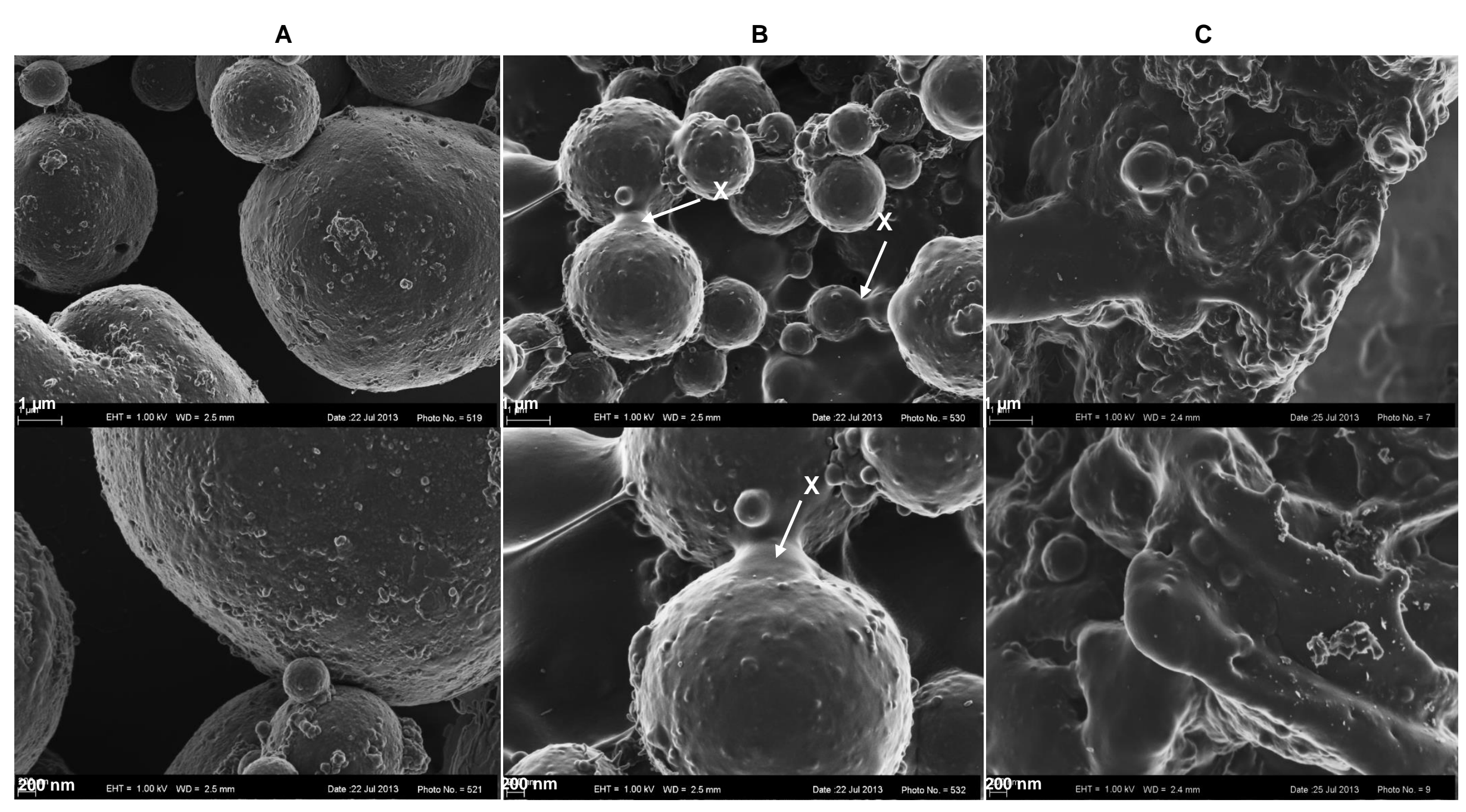

Figure 4 
A

$\mathrm{kDa}$

MW

\section{A}
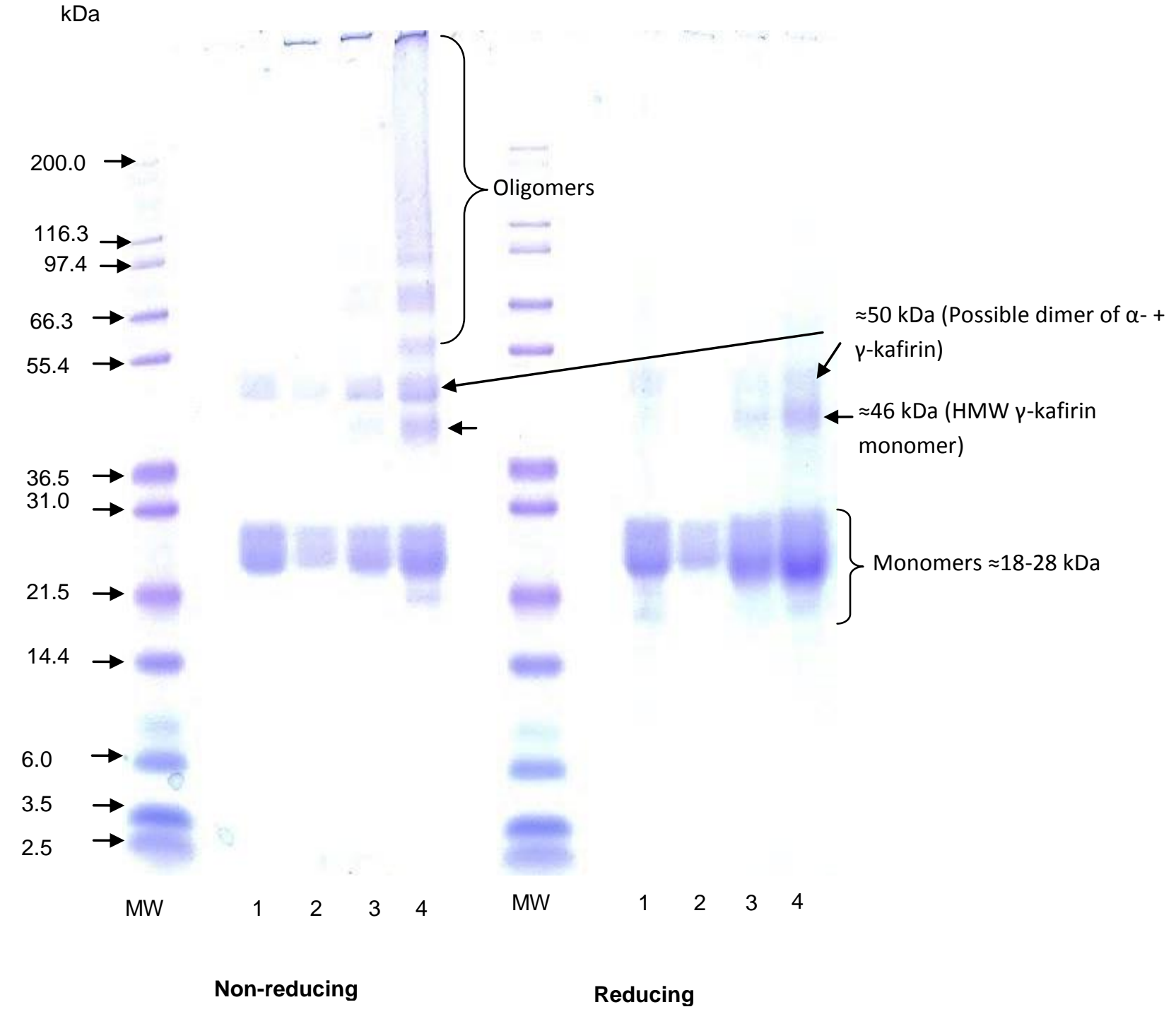

B

Figure 5 

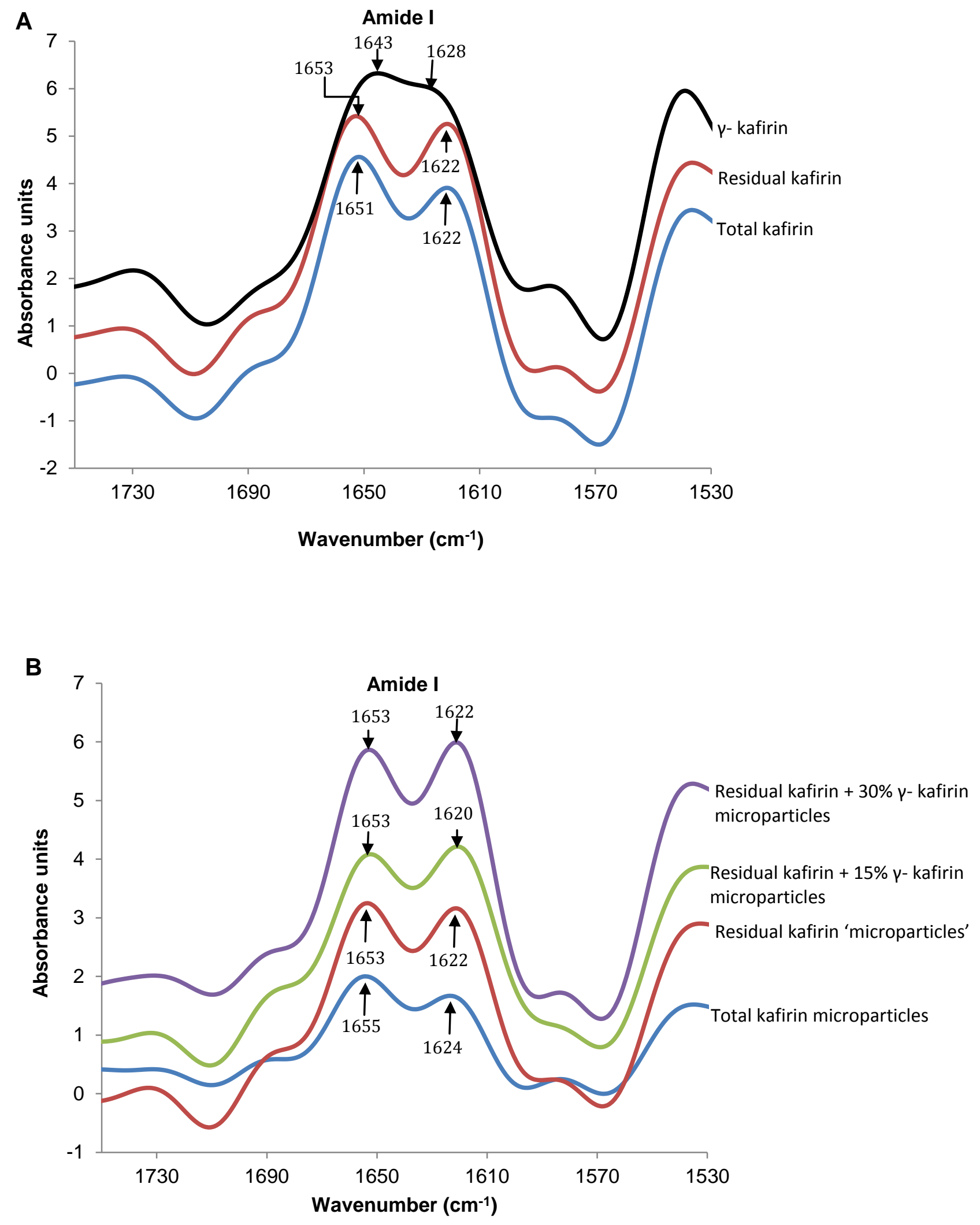

Figure 6 


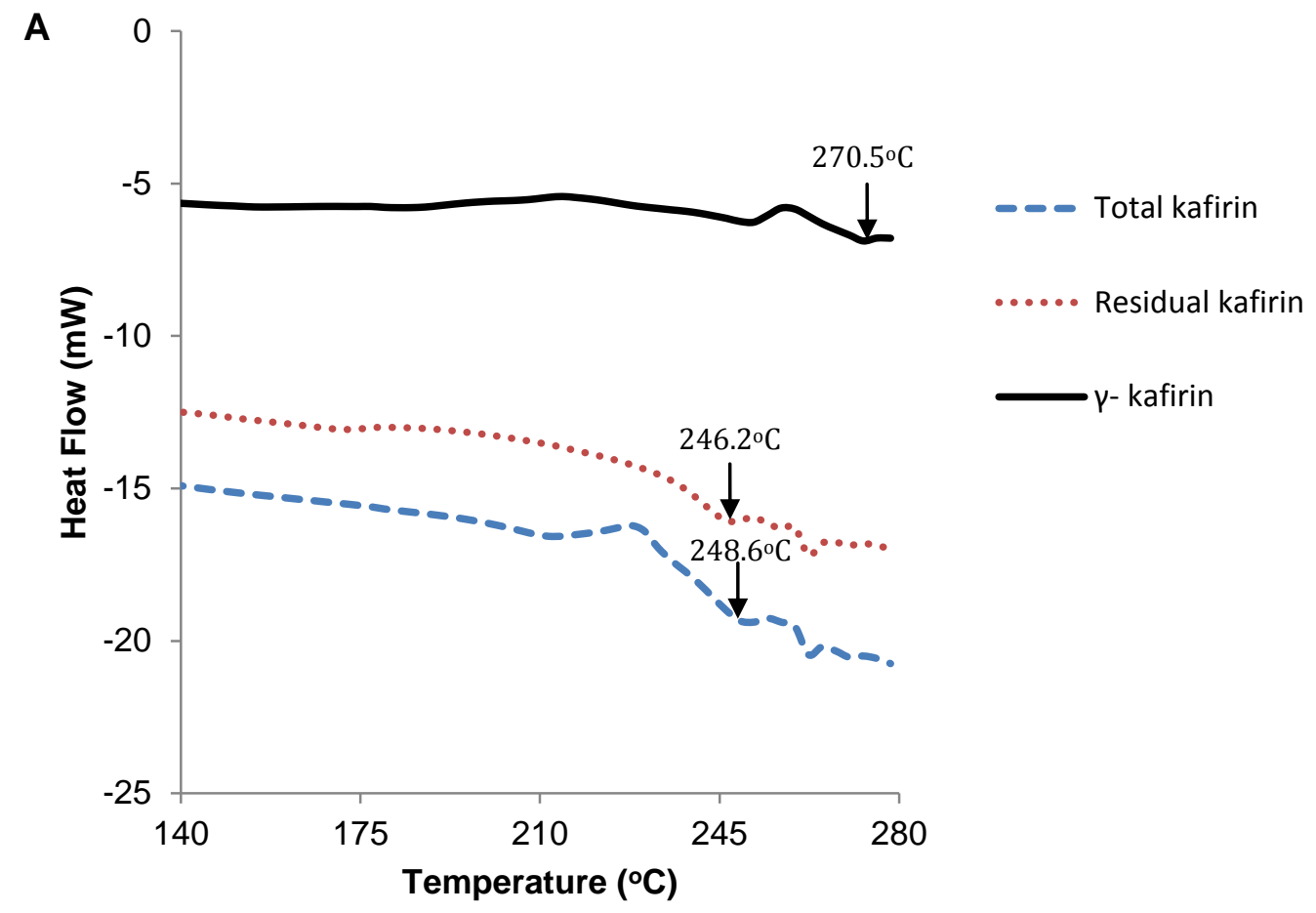

B

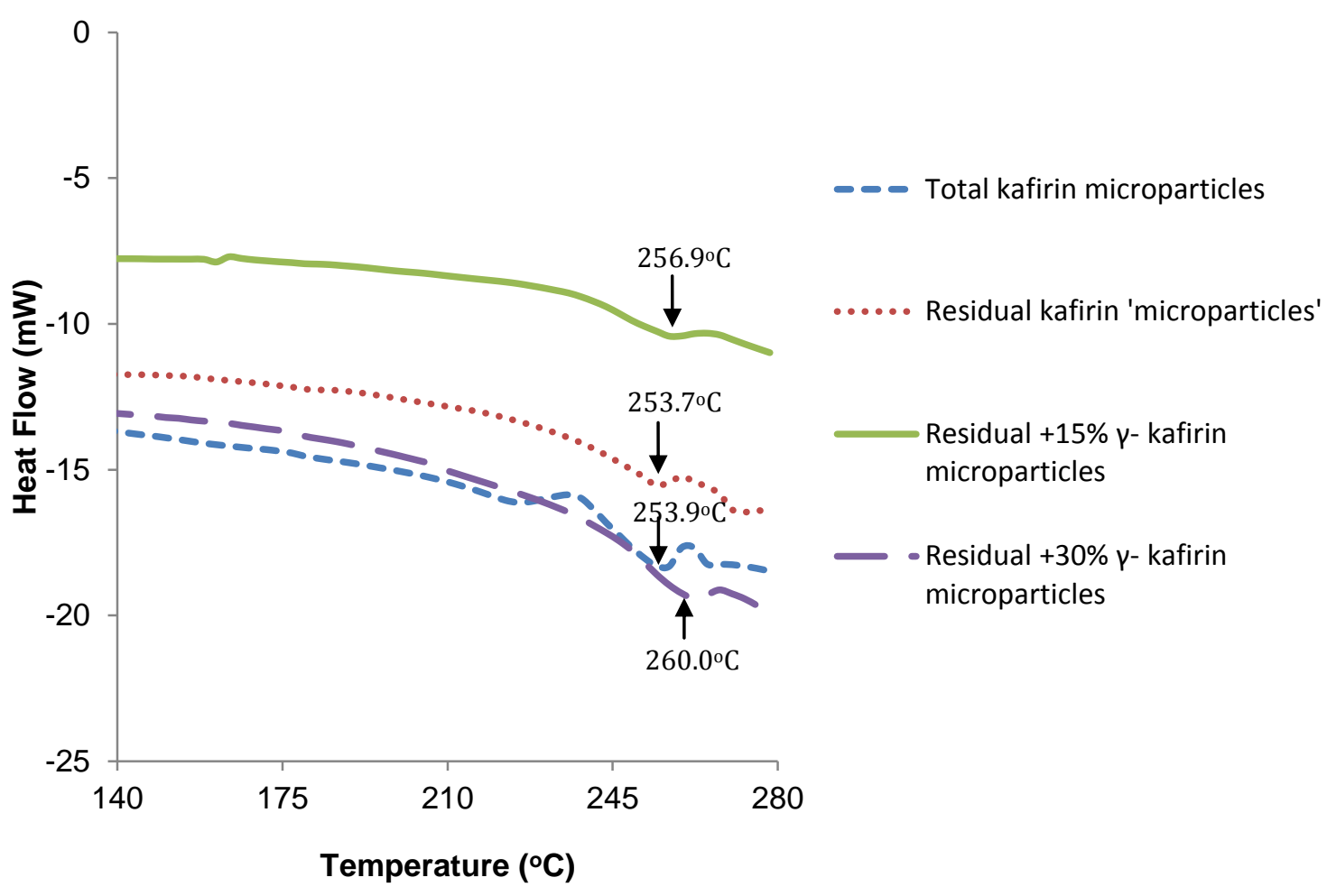

Figure 7 


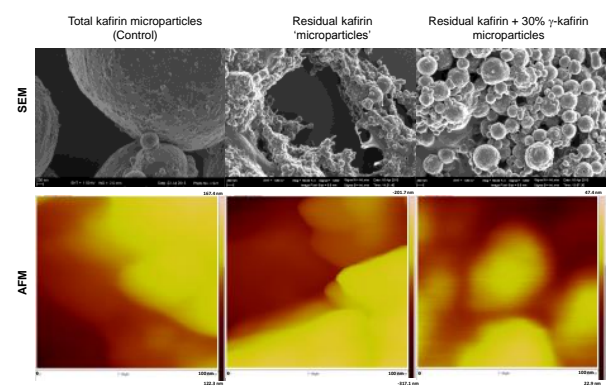

Toc Graphic 